\title{
Reaction Kinetics in Restricted Spaces
}

\author{
RaOUl Kopelman* and Yong-Eun Koo \\ Department of Chemistry, University of Michigan, Ann Arbor, MI 48109, USA
}

(Received 2 August 1990)

\begin{abstract}
Reactions in restricted spaces rarely get stirred vigorously by convection and are thus controlled by diffusion. Furthermore, the compactness of the Brownian motion leads to both anomalous diffusion and anomalous reaction kinetics. Elementary binary reactions of the type $\mathrm{A}+\mathrm{A} \rightarrow$ Products, $\mathrm{A}+\mathrm{B} \rightarrow$ Products, and $\mathrm{A}+\mathrm{C} \rightarrow \mathrm{C}+$ Products are discussed theoretically for both batch and steady-state conditions. The anomalous reaction orders and time exponents (for the rate coefficients) are discussed for various situations. Global and local rate laws are related to particle distribution functions. Only Poissonian distributions guarantee the classical rate laws. Reactant self-organization leads to interesting new phenomena. These are demonstrated by theory, simulations, and experiments. The correlation length of reactant production affects the selfordering length scale. These effects are demonstrated experimentally, including the stability of reactant segregation observed in chemical reactions in one-dimensional spaces, e.g., capillaries and microcapillaries. The gap between the reactant A (cation) and B (anion) actually increases in time and extends over millimeters. Excellent agreement is found among theory, simulation, and experiment for the various scaling exponents.
\end{abstract}

\section{INTRODUCTION}

Chemical kinctics in low dimensions usually involves active sites or surfaces which lower significantly the activation energy barriers. At the same time, there is essentially no convective stirring in low dimensions and the diffusion process is less efficient due to the compactness $^{1}$ of the Brownian motion. As a result, low-dimensional reactions are very often diffusion-limited. Furthermore, the so-called compact Brownian motion leads to anomalous diffusion ${ }^{2}$ and to anomalous rate laws. ${ }^{3}$ Finally, these anomalous macroscopic rate laws are a manifestation of the unexpected entropic self-ordering of reacting molecules, i.e., a dramatic self-organization on a microscopic or mesoscopic (and sometimes macroscopic) length scale. ${ }^{4}$

Practically every heterogeneous reaction is a lowdimensional reaction, whether it occurs on a surface, inside a pore, at a micelle, or elsewhere. In addition, some homogeneous reactions, such as solid-state reactions, occur preferentially in low-dimensional environments, e.g., domain boundaries, defect sites, surface steps, or radiation tracks. Furthermore, reactions such as polymerization, gclation, and ccllular growth have a low-dimensional dynamics. We also note that mechanistically, these low-dimensional reactions may involve molecules, radicals, atoms, clectrons, holes, excitons, or solitons.

Experimentally, simple elementary reactions that have been observed to follow strange rate laws range from solution photodimerization inside porous polymeric membranes ${ }^{5,6}$ to exciton fusion in molecular alloys. ${ }^{3}$ Further evidence for such anomalies comes from excimer kinetics in polymer blends, ${ }^{7-9}$ oxygen quenching in porous media, ${ }^{10}$ energy transfer in photosynthetic systems, ${ }^{11}$ and neuron gating kinetics in sodium channels. ${ }^{12}$ There also appear to be many other instances where anomalous rate laws have been fitted (unnecessarily) by complex mechanisms.

There are three major categories of simple, elementary, bimolecular mechanisms:

$$
\begin{gathered}
A+A \rightarrow \text { Products } \\
A+B \rightarrow \text { Products } \\
A+C \rightarrow C+\text { Products. }
\end{gathered}
$$

*Author to whom correspondence should be addressed. 
In the above we assume that the products are inert or leave the reaction medium (e.g., desorb). We also assume that in reaction 2 there is no $\mathrm{A}+\mathrm{A}$ or $\mathrm{B}+\mathrm{B}$ reaction mechanism. We note that in reaction $3, C$ is a catalyst or catalytic site. Reactions 1 and 2 are described by the textbook bimolecular rate equations and reaction 3 by the pscudomonomolecular equation. We also note that reaction 1 is second-order in A concentration while reaction 2 is second-order overall, i.e., first-order in A and first-order in B. Reaction 3 is actually first-order in both $A$ and $C$ (if $C$ is varicd from case to case) and thus also second-order overall, while being pseudo-first-order in a given case (where only A varies in time). It turns out that these thrce categories (1-3) give rise to extremely different rate laws for low dimensions. Furthermore, within cach category there arise various subcategories with drastically different rate laws, depending on distinctions such as dimensionality, initial conditions, source (input) conditions, and other subtle constraints which do not affect the textbook rate laws. Even the achievement of a steady state is no longer guaranteed by a steady input of particles. The new "zoo" of rate laws is demonstrated by a few examples of reactions constrained to one dimension (Table 1). While some early theoretical work and some early experiments may have indicated rate law anomalics, ${ }^{13,14}$ it was the development of computer simulations that became the major force behind the recent developments in this area.

The importance of short-time fluctuations has alrcady been pointed out by Einstein in his 1905 papers on Brownian motion. However, the possibility of drastic long-time fluctuations was probably first pointed out by the famous Sovict chemist, physicist and astrophysicist, Zcldovich. ${ }^{15}$ Similar long-time fluctuation phenomena have been pointed out in the context of solid-state kinetics. ${ }^{16}$ It is the occurrence of such long-time and long-range fluctuations that leads to reactant self-ordering and to the strange new rate laws for elementary reaction mechanisms.

We first review the theory and simulations from the standpoint of an experimentalist, emphasizing the nature and form of the novel laws. We also attempt to give physicochemical rationalizations for and insights into this new paradigm. We then describe some selected experiments in order to demonstrate these laws and the consequences and opportunities which they create.

\section{THEORY AND SIMULATIONS}

\subsection{Batch Reactions}

These "big bang" reactions were the first to be treated with respect to the long-time, long-range "fluctuations" that give rise to the anomalous asymptotic (long-time) behavior. For instance, ${ }^{17,18}$ the "trapping reaction" and the "target reaction" are both special cases of reaction 3:

$$
\mathrm{A}+\mathrm{C} \rightarrow \mathrm{C} \text {. }
$$

In the trapping reaction $C$ is fixed and $A$ moves, while in the target (quenching) reaction $A$ is fixed and $C$ is mobile. Classically, one expects the survival function $C_{\mathrm{A}}$ (A concentration) to decrease exponentially in time:

$$
C_{\mathrm{A}}=C_{\mathrm{A}}^{0} \exp (-k t),
$$

where $C_{\mathrm{A}}^{0}$ is the "big bang" $(t=0)$ reactant concentration and $k$ the rate constant. In the Smoluchowski treatment of diffusion-limited reactions, ${ }^{17} k-D$ where $D$ is the diffusion constant. However, this is only valid in three (or higher) dimensions. For instance, the Smoluchowski approach in one dimension leads to a different asymptotic solution:

$$
C_{\mathrm{A}} \sim \exp \left(-k^{\prime} t^{\prime 2}\right)
$$

Furthermore, taking fully into account the long-range fluctuations leads to ${ }^{16}$

$$
C_{A}^{\prime} \sim \exp \left(-k^{\prime \prime} t^{1 / 3}\right)
$$

Many such new relations have been derived ${ }^{16,18}$ for low (including fractal) dimensions.

Replacing eqs $1-3$ by

$$
A+i=P, \quad i=A, B, C,
$$

where $\mathrm{P}$ is any resulting products (including $\mathrm{A}$ and/or $\mathrm{i}$ ), a simple way of describing many of the results is to consider the differential rate equation (rather than the integrated forms, involving the "survival function" $C_{A}$ ):

$$
-\left(d C_{\mathrm{A}} d d\right)=k(\imath) C_{\mathrm{A}} C_{\mathrm{i}}, \quad \mathrm{i}=\mathrm{A}, \mathrm{B}, \mathrm{C} .
$$

The nonclassical results can now be written (for long times, $t \rightarrow \infty$ )

$$
k(t) \sim t^{-h}, \quad h<1,
$$

with the special case $h=0$ for the classical (textbook) case. In particular, for low dimensions $(d<2)$, one finds ${ }^{19}$ for certain $\mathrm{A}+\mathrm{A}$ reactions:

$$
h=1-d_{3} / 2 \text {, }
$$

while for the same $\mathrm{A}+\mathrm{B} \rightarrow 0$ reactions, ${ }^{20}$

$$
h=1-d_{3} / 4
$$

whenever the spectral (fracton) ${ }^{21}$ dimension $\left(d_{3}\right)$ is $d_{3}<4$.

Another simple, way of describing many of the results, and particularly those for stcady-state reactions, is: 


$$
-d C_{\mathrm{A}} / d t=k C_{\mathrm{A}}^{y} C_{\mathrm{i}}^{z},
$$

where $k$ is a time-independent constant and the partial orders $y$ and $z$ may be nonintegers, and the same is true for the overall order

$$
x=y+z .
$$

We emphasize that all this is for an elementary bimolecular reaction, where the molecularity of the reaction is described by eq 8 . Obviously, by the classical (textbook) reaction laws, $x, y$, and $z$ are all integers $(y=1, z=1$, $x=2$ ). For instance, for $A+A$ reactions on a critical percolation cluster $x=5 / 2$, for a Sierpinski gasket it is $x=2.47$ and, in general, it is $x=1+2 / d_{\mathrm{g}}$. Similarly, this empirical form (eqs 12,13) accounted for the strange results listed in Table 1.

Table 1. Reaction Orders $x$ in One Dimension

\begin{tabular}{lcc}
\hline & \multicolumn{2}{c}{$x$} \\
\cline { 2 - 3 } Reaction & $\begin{array}{c}\text { Steady } \\
\text { state }\end{array}$ & $t \rightarrow \infty$ \\
\hline Random $A+A \rightarrow A_{2} \uparrow$ & 3 & 3 \\
$\downarrow A_{2} \rightarrow A+A \rightarrow A_{2} \uparrow$ & 2 & 3 \\
Classical bimolecular & 2 & 2 \\
Random $A+B \rightarrow A B \uparrow$ & $4^{\text {a }}$ & 5 \\
$\downarrow A B \rightarrow A+B \rightarrow A B \uparrow$ & 2 & 3 \\
If $A+A \rightarrow A_{2} \uparrow$ and $B+B \rightarrow B_{2} \uparrow$ & 3 & 3 \\
Examples: hydrogen on Pt wire & & \\
$\downarrow H_{2} \rightarrow H+H \rightarrow H_{2} \uparrow$ & 2 & 3 \\
$\downarrow D_{2} \rightarrow D+D \rightarrow D_{2} \uparrow$ & 2 & 3 \\
$H+D \rightarrow H D \uparrow$ & 3 & 3 \\
\hline
\end{tabular}

${ }^{\mathrm{a}}$ For hard-sphere particles and vertical reactions; otherwise $x=2$.

In the last few years there has been a bewildering explosion of new results. It would be impossible to list them all, let alone point out problems and contradictions. We list a few papers that include partial revicws $s^{4,22,23}$ and give below what we consider to be the main problems of interest.

The beauty of classical kinetics lies in the universality of its formulation. The functional form of the rate laws does not depend on the dimension or dimensionality of the medium, initial conditions (for batch reactions), details of source (for steady-state reactions), nature of motion (ballistic, diffusion), relative mobility and reaction of the components, etc. Such factors only affect the values of the parameters. It is also implied that global and local rate laws are the same and that the particle distribution functions depend only on the concentrations, but not on whether the ensemble is at equilibrium or far from it, or at steady state or far from it.

When deviations from classical behavior are studied, it is important to distinguish the various factors behind such deviations and the interactions among them. Often it is only with a combination of two such factors that one finds deviations from the classical picture, e.g., with a combination of low dimensionality and a random source term (but not with low dimensionality and a "geminate" source term).

Nonclassical diffusion-controlled reaction kinetics does retain scaling and universality relations. ${ }^{24-26} \mathrm{How}$ ever, they differ in detail for different kinds of reactions $(A+A$ vs. $A+B)$, for different modes of reactions (steady state vs. batch), for different conditions (source term structure, initial distributions), for different conservation laws (exact vs. statistical equality of reactant species), etc. To get a better insight into the class structure of such scaling laws is one problem of interest to us.

Global rate laws have been of much interest. They indeed were the first targets of modem investigations. However, it has become apparent that there are instances where precise global laws do not exist. Can they be substituted by local laws? Can one always define a local law? What is the relation between a local and a global law? This is another problem of interest to us.

Distribution functions have been the linchpins of statistical mechanics. They have also been recognized as the basic information required for the qualitative and quantitative understanding of reaction kinetics. ${ }^{4,27}$ Probably the most stringent test of any kinetic theory is the goodness of the distribution functions predicted by it. Computer simulations are adept at testing distribution functions with almost as much ease as testing rate laws. ${ }^{27.28}$ Examples ${ }^{29}$ are given in Figs. 1 and 2. The distribution functions can also serve as a bridge between local and global rate laws (to the extent that they exist). Furthermore, on an empirical basis, computer simulations of distribution functions show trends and clues that may be useful for the design of improved theories. Such trends may also be of much aid to experimentalists, providing new qualitative or intuitive model pictures. For instance, the buildup of depletion zones around reactants or catalytic sites depends on specific parameters such as medium dimensionality and reactant mobility. ${ }^{30,31}$ Recently there has been much progress in the area of analytical models for diffusion-controlled reaction kinetics. ${ }^{32,33}$ 


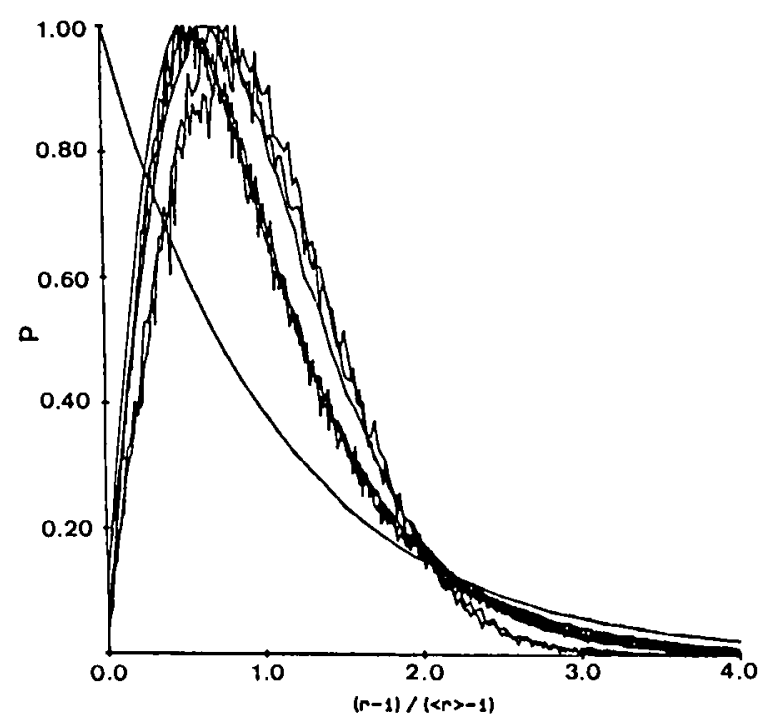

Fig. 1. Normalized distributions of nearest-neighbour distances $r$ for one-dimensional kinetics. ${ }^{29}$ The normalization of $r$ is done by calculating the quantity $(r-1) /(<r\rangle-1)$, where $<r>$ is the average gap distance for all particles at any given time. Lattice size is $L=10000$ sites, initial density is 0.05 . The exponential single curve is the initial $(t=0)$ Poissonian distribution; the two groups of curves are for the $\mathrm{A}+\mathrm{A} \rightarrow 0$ reaction (left) and $\mathrm{A}+\mathrm{A} \rightarrow \mathrm{A}$ reaction (right); in each group the distribution is shown for different reactions times: $t=100,1000$, and 2000 . All curves are averages of 5000 runs $(A+A \rightarrow 0)$ and 1000 runs $(A+A \rightarrow A)$.

\subsubsection{A + A Reactions}

This is apparently the simplest and best understood class of reactions. Somewhat surprisingly, the coagulation reaction

$$
\mathrm{A}+\mathrm{A} \rightarrow \mathrm{A}
$$

is better understood than the annihilation reaction,

$$
\mathrm{A}+\mathrm{A} \rightarrow 0 \text {. }
$$

Even more surprising are the drastic differences between these two, in particular the difference in the particle distribution functions. This difference first became apparent from our simulations on interparticle distribution functions. ${ }^{4.28}$ It was elegantly proven in the recent analytical work of Doering and Ben-Avraham. ${ }^{32}$ Specifically, in one dimension, the interparticle distance distribution probability $P(r)$ was found to have the form:

$$
P(r)=(\pi / 2) C r \exp \left[-(\pi / 2) C^{2} r^{2} / 2\right]
$$

for long times, where $C=C(t)$ is the instantaneous density and $r$ is the interparticle distance. (The closely related nearest-neighbor distance distribution is shown in

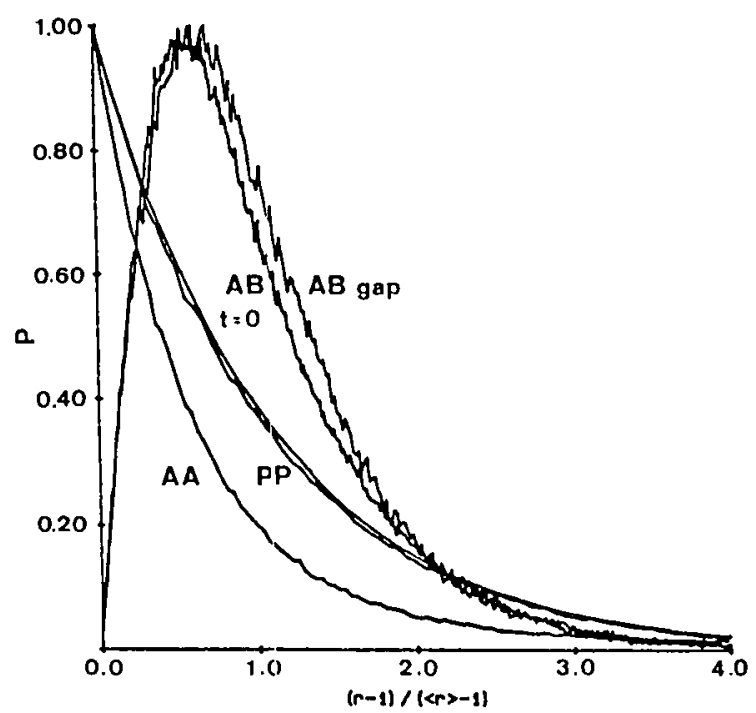

Fig. 2. Nearest-neighbor distance distributions (NNDD) for one-dimensional $\mathrm{A}+\mathrm{B} \rightarrow 0$ reactions. ${ }^{29}$ From the left, the three exponential-type curves are NNDD for $t=1000$ likelike (AA), for $t=1000$ particle-particle (PP), and for $t=0$ (same Poissonian distribution for all cases, i.e., AA, BB, and $\mathrm{PP})$, respectively. The two curves with a well-developed maximum are $t=1000$ like-unlike (AB) NNDD (left) and like-unlike (AB) gap (interparticle distance) distribution (right). All NNDD curves are averages of 2000 runs and the gap curve is that of 5000 runs. Total number of lattice sites is 10000 and the initial $(t=0)$ concentration is 0.05 for $\mathrm{A}$ and 0.05 for $\mathrm{B}(0.1$ for $P)$.

Fig. 1 based on simulation results). The first important observation is that this distribution differs drastically from the Poissonian distribution (in one dimension):

$$
P(r)=C \exp (-C r) \text {. }
$$

The "maximum" of the Poissonian distribution is at $r=$ 0 . The kinetic distribution has a minimum there (with $P(0)=0)$. The maximum of the kinetic distribution is at $r=(2 / \pi)^{1 / 2} C^{-1}$. Obviously this maximum increases with time, i.c., as the density decreases (this is evidently not so for the Poissonian distribution). Furthermore, for large values of $r$, the kinetic distribution has a Gaussian tail (while the Poissonian distribution has an exponential one). We also note that for small values of $r$, the kinetic distribution increases linearly with $r$, while the Poissonian one decreases exponentially. As the reaction kinetics is mainly determined by the small $r$ range, the resulting rate law is cubic in $C$, while with a Poissonian distribution it is quadratic in $C$ (the classical result). This is consistent with the older Torney and McConnell ${ }^{34}$ result (with the Anacker et al.$^{35}$ transformation). 
We now turn to the annihilation reaction $(\mathrm{A}+\mathrm{A} \rightarrow$ 0 ). There is no analytical result, so far, for one dimension. However, there is an asymptotic analytical result, ${ }^{36}$ for $r \rightarrow \infty$ : the tail of the distribution has an exponential, rather than Gaussian form. (This is also seen from the simulation results in Fig. 1.) This is a major distinction. However, the small $r$ part of the distribution is linear, as in the coagulation $(A+A \rightarrow A)$ case, thus giving a similar cubic rate law. On the other hand, the central part (intermediate $r$ ) already differs from the coagulation case, and the maximum falls at about $0.4 C^{-1}$ (half the value of the coagulation case). A rough approximation ${ }^{28,29}$ is

$$
P(r) \sim C r \exp [-\alpha C r],
$$

where $\alpha$ is a coefficient that has not been determined from simulations, due to the poor quality of the functional fit. ${ }^{29}$

\subsection{2. $A+B \rightarrow$ O Reactions}

We imply that there occur no $\mathrm{A}+\mathrm{A}$ or $\mathrm{B}+\mathrm{B}$ reactions (otherwise this case reverts to the $\mathrm{A}+\mathrm{A} \rightarrow 0$ reaction). This is the Zeldovich ${ }^{15}$ case, which essentially was the start of modern diffusion-reaction theory. This model assumes a random initial $(t=0)$ distribution, with random fluctuations. Globally, $C_{\mathrm{A}}=C_{\mathrm{B}}=C / 2$ at $t=0$ and at every other instant in time. However, locally, $\Delta C$ $=C_{\mathrm{A}}-C_{\mathrm{B}}$ fluctuates around zero at $t=0$. His formalism shows that $2 \mathrm{l} \triangle C \mathrm{I} / \mathrm{C}$ increases in time and becomes of the order of unity at $t \rightarrow \infty$, provided that the dimension of the reaction space is $d<4$. This means that a segregation of reactants proceeds in time. A very small segregation $(2|\Delta C| / C \ll 1)$ at $t=0$ turns into a large one $(-1)$ at $t \rightarrow \infty$. Concomitantly, the classical survival probability

$$
C=2 C_{\mathrm{A}}=2 C_{\mathrm{B}}-t^{-1}, t \rightarrow \infty
$$

is valid only for high dimensions $(d>4)$ but is replaced by

$$
C-t^{-d / 4}, t \rightarrow \infty, d<4
$$

for all "practical" dimensions $(d \leq 3)$. This formalism was generalized somewhat (and proved numerically) by Toussaint and Wilczek, ${ }^{37}$ and generalized to fractals by Kang and Redner ${ }^{20}$ (replacing $d$ by $d_{s}$, the "spectral" dimension).

In the above discussions it was implied that $\mathrm{A}$ and $\mathrm{B}$ have identical mobilities. The other extreme case is where one of them (say B) is immobile ("sitters"). This case, as well as the intermediate cases, has been studied by Zumofen et al. ${ }^{22,38}$ with some interesting results. It is intuitively obvious that complete spatial segregation is harder to achieve and will take longer. Quantitatively, it is not yet clear how the particle distributions depend on the relative mobilities of the two species.

Toussaint and Wilczek ${ }^{37}$ have also argued that for a correlated $t=0$ distribution (e.g., geminate AB pairs), the asymptotic rate law is different:

$$
C \sim t^{-d 2}, d \leq 2, t \rightarrow \infty,
$$

i.e., the same as for $\mathrm{A}+\mathrm{A}$ reactions, ${ }^{3,20}$ and that there is no segregation. It turns out ${ }^{39}$ that the situation is more complex and eq 21 is only correct for strictly geminate conditions. For correlated but nongeminate initial conditions (where the correlation length is much larger than the particle size):

$$
\begin{array}{ll}
C-t^{-(-d+2) / 4}, & d \leq 2, t \rightarrow \infty \\
C-t^{-1}, & d \geq 2, t \rightarrow \infty .
\end{array}
$$

Finally we note that there are no published experiments showing self-organization or anomalous kinetics for A + $B$ reactions.

\subsection{3. $A+C \rightarrow C$ Reactions}

The early work for this case has centered around "trapping" reactions, i.e., where A diffuses and C is fixed in space. This work also originated in the Soviet Union and is summarized, in part, in the monograph of Agranovich and Galanin. ${ }^{16}$ The typical rate law in one dimension was given in eq 6 and was rationalized by a long-time distribution of particles away from the traps. The first exact (analytical) work on particle distributions appears to be that by Weiss et al. ${ }^{30}$ It is for a single trap $\mathrm{C}$ in three- and one-dimensional continua of particles $\mathrm{A}$ (with density $C_{\mathrm{A}}$ ). Again the nearest neighbor ( $\left.\mathrm{C}-\mathrm{A}\right)$ distance distribution is far from randorn in one dimension (while essentially random in $d=3$ ). Actually, the distribution function is quite similar to that for $\mathrm{A}+\mathrm{A} \rightarrow$ A (eq 16) in one dimension. Similarly, also, the global rate law is, in one dimension,

$$
\left|d C_{\mathrm{N}} d t\right|-t^{-1 / 2} C_{\mathrm{A}},
$$

while in three dimensions the classical result is recovered:

$$
\left|d C_{\mathrm{A}} / d t\right| \sim C_{\mathrm{A}},
$$

This is again similar to the $A+A$ case. We note that eqs 22 and 23 are the first exact results for the original Smoluchowski problem ${ }^{17}$ of accretion of molecules by a colloidal particle (or a crystallization seed). Smoluchowski's solution for the three-dimensional case is indeed equivalent to eq 23. 


\subsubsection{Self-Ordering}

In cach of the three above cases one finds deviations from uniformly random particle distributions, deviations that increase with time. Often it requires only a few time "steps" (in the simulation or the discrete model) to attain a drastically different (e.g., "Wigner-like"4.28 distribution. This spontaneous self-ordering, based totally on diffusion and reaction (but no long-range forces) is of major interest. There are similarities ${ }^{29}$ in the "patterns" of different reaction classes $(A+A$ vs. $A+B)$ and dissimilarities ${ }^{4,29}$ in those of very similar reactions ( $A+A$ $\rightarrow 0$ vs. $A+A \rightarrow A)$. Some of the patterns take much longer to form than others. Dimensionality and initial conditions are also of prime importance. The investigation of such patterns requires some new tools (e.g., order parameters). For instance, we have defined ${ }^{27.40}$ about a dozen different "segregation parameters." The relative usefulness of such order parameters in different situations is also of some importance. Particle distributions or partial particle distributions, as well as quantities derived from them, can also be used as such tools.

\subsection{5. $A+B \rightarrow C$ Where $A$ and $B$ Are Segregated throughout the Reaction}

Recently, analytical work on an $\mathrm{A}+\mathrm{B} \rightarrow \mathrm{C}$ type reaction-diffusion process in an effective one-dimensional system has been done by Galfi and Racz. ${ }^{41}$ The reactant A of constant density $a_{0}$ and $\mathrm{B}$ of constant density $b_{0}$ are initially separated. They meet at time 0 , forming a single reaction boundary, which makes the system effectively one-dimensional. The motion of the reaction front with time is shown in Fig. 3. This model is similar to that of Weiss et al. ${ }^{30}$ for $\mathrm{A}+\mathrm{C} \rightarrow \mathrm{C}$, where $\mathrm{A}$ is a one-dimensional continuous solute and $C$ is a single trap. However, here both $\mathrm{A}$ and $\mathrm{B}$ are continuous (and $\mathrm{A}$ and $\mathrm{B}$ are like traps to each other). The results from the set of the reaction-diffusion equations for $\mathrm{A}$ and $\mathrm{B}$, which are valid in the long-time limit, show that $x_{\mathrm{f}}$ (the position of the center of reaction front) scales with time as $x_{\mathrm{f}}-t^{1 / 2}$, while $w$ (the widih of the reaction front) scales as $w-t^{1 / 6}$ and the production rate $r_{\mathrm{f}}\left(\right.$ at $\left.x=x_{\mathrm{f}}\right)$ scales as $t^{-2 / 3}$. We can also find that the global reaction rate $R$ scales as $t^{-1 / 2}$, i.e.,

$$
R=d C / d t \sim t^{-1 / 2}
$$

This is similar to the results of Weiss et al.'s work ${ }^{30}$ for $\mathrm{A}+\mathrm{C} \rightarrow \mathrm{C}$ (eq 22 for $d A / d t)$.

\subsection{Steady Source Reactions}

Steady source reactions are of prime importance in biology, ecology, geology, technology, and many other areas. ${ }^{4}$ They also give a wider range of phenomena due to three factors: (1) the richness of source-term structures (sce below); (2) the inclusion of strange topologies (e.g., "dust" or "fractal dust") which would not support batch reactions for long; (3) ordering phenomena that for batch reactions are only achievable at extremely long times, i.e., at vanishing densities, but under steady source conditions may develop even at high densities.

\subsubsection{Source-Term Structures}

The most common source term is uniformly random in both space and time. One of the surprising results has been the fact that the mere presence of such a source, combined only with diffusion and reaction, may lead to highly self-ordered systems. ${ }^{4,42}$ Another common source produces particles correlated in space andlor time. For instance, when $\mathrm{H}_{2}$ particles land on a platinum surface they dissociate into pairs of atoms; or when electrons and holes are produced by photons hitting a photovoltaic (a)

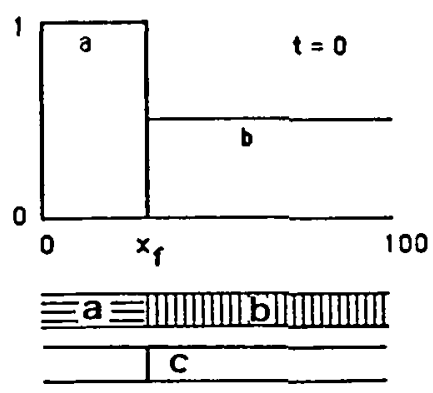

(b)

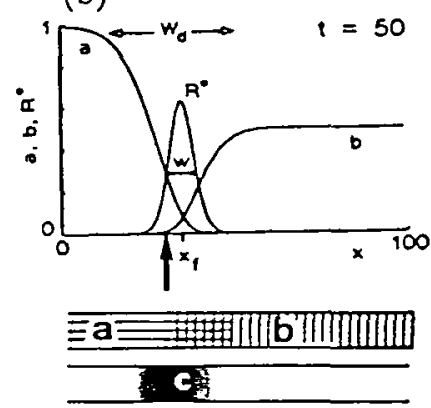

(c)

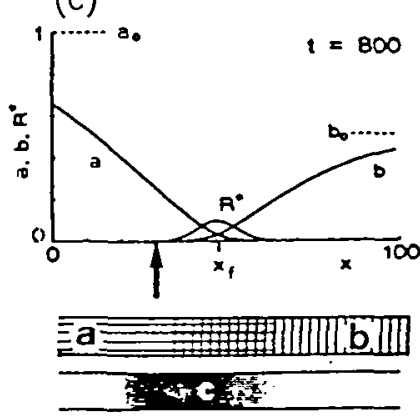

Fig. 3. The motion of the reaction front with time. Length $(x)$, time $(t)$, the densities $(a, b)$ of the reagents (A, B), and the magnified production rate $\left(R^{*}=100 R=100 \mathrm{kab}\right)$ of $\mathrm{C}$ are scaled to be all dimensionless. As an initial condition, we used: $a=1, b=0$ for $x<30$, and $a=0, b=0.5$ for $x>30$ (the $t=0$ position of the center of the front, $x_{\mathrm{f}}$, is shown by an arrow). The top figures of (b) and (c) are from ref 41. 
cell, they are produced geminately. Alternatively, the source may be a hot beam of dissociated $A B$ molecules, landing as pairs of $\mathrm{A}$ and $\mathrm{B}$ atoms on a surface, correlated in time but not in space. The particles may be able to react as they land on top of other particles (vertical reaction) or they may not, depending on the chemistry or physics of the situation. If they are not able to react, they may be discarded or they may land in the immediate vicinity, depending again on the particular reaction. Sources with vertical reaction may produce particle distributions that differ drastically from those produced with no vertical reaction. Also, the atoms on a surface may occasionally desorb, and the excitons produced in a solid have a natural decay time, independent of the annihilation or fusion reaction. Such a "monomolecular" (first-order) decay process adds another random (negative) contribution to the source terms. All such possible source structures have significant effects on the outcome, in addition to the well-known source-term distinctions, such as an unevenness in A and B production terms (alternatively viewed as a strong $A$ source and a weaker B source). Furthermore, initial conditions may vary, such as an initially empty lattice (the usual case) vs. a fully or partially occupied one. We also note that the source structure usually determines the conservation laws. For instance, a time-correlated, pairwise production of $A$ and $B$ particles (in an $A+B \rightarrow 0$ reaction with no decay) will guarantee an exact global conservation of the equality $C_{\mathrm{A}}=C_{\mathrm{B}}$. On the other hand, a geminate production of $\mathrm{A}$ and $\mathrm{B}$ will guarantee both an exact global and an exact local conservation of $C_{\mathrm{A}}=C_{\mathrm{B}}$ (i.e., no segregation). However, a uniformly random source term gives only a statistical global conservation of $C_{\mathrm{A}}=$ $C_{\mathrm{B}}$, and, under certain circumstances (e.g., no desorption), this may lead to long-time realizations with $C_{\mathrm{A}} \gg$ $C_{\mathrm{B}}$ or vice versa.

An obvious question of interest is whether a given source term will produce a steady-state system. The answer may depend on a combination of source structure and medium dimensionality, or a combination of other conditions. There have been several recent surprises in this respect, ${ }^{23-26,43}$ which lends importance to future work aimed at clarifying this issue. If a steady state is achieved, the next question is: is the particle distribution uniformly random or ordered? The self-ordering process itself may either hinder or aid the generation of a steady state. The self-ordering is, therefore, an interesting related question.

Certain source terms may generate a steady state that mimics an equilibrium state. For instance, strictly geminate pair creation, with an annihilation reaction $(A+A$ $\rightarrow 0$ or $\mathrm{A}+\mathrm{B} \rightarrow 0$ ), is expected to set up a steady state that mimics equilibrium. We expect equilibrium states to be totally (randomly) disordered (by the second law of thermodynamics). Such source terms and steady states are thus of special interest. However, the steady states which are self-ordered (i.e., far from equilibrium) are of even more interest. It is they who are responsible for the anomalous reaction rate laws. They may even lead to much speculated upon phenomena, such as biomorphogenesis, ${ }^{23,24}$ which are in apparent (though not real) contradiction with the second law.

\subsubsection{A + A Reactions}

Analytical solutions exist so far only for a limited number of cases and are restricted to one dimension. For the $\mathrm{A}+\mathrm{A} \rightarrow 0$ reaction, $\operatorname{Racz}^{44}$ has solved "one and a half" cases. For the geminate landing case the solution is complete: for the steady-state reaclion the rate law is classical (second-order) and the particle distribution is uniformly random. This is actually expected for all simple geminate landing cases, because, as pointed out above, such steady states map onto equilibrium states,

$$
A+A \leftrightarrow A_{2},
$$

and for equilibrium states we expect this classical behavior. On the other hand, for uniformly random landing, where a steady state is also achieved, the rate law is third-order in density. Furthermore, the particle distribution is nonrandom. However, $\mathrm{Racz}^{44}$ did not derive a functional form for such a distribution.

A recent paper by Doering and Ben-Avraham ${ }^{33}$ gives a similar derivation for the one-dimensional $\mathrm{A}+\mathrm{A} \rightarrow \mathrm{A}$ problem. Again a steady state is found for the random (and geminate?) source terms. The rate law (for the random source) is again third-order in density, and the particle distribution is nonrandom. In addition, the interparticle distance distribution was derived specifically (in terms of Airy functions).

Simulation-based results were obtained for all dimensions (or spectral dimensions, for fractals), both higher and lower than unity. Equations 12 and 13 actually give, for irreversible $A+A$ reactions,

$$
-d C_{N} d t=k C_{\mathrm{A}}^{\mathrm{x}}+R,
$$

where $R$ is the source term. As mentioned above, for these $\mathrm{A}+$ A reactions,

$$
\begin{array}{ll}
x=1+2 / d_{3}, & d_{3}<2 \\
x=2, & d_{1}>2,
\end{array}
$$

while for the marginal (critical dimension $d=2$ ), one indeed obtains $x=2$ but a logarithmic correction appears: 


$$
-d C_{\mathrm{A}} / d t=k C_{\mathrm{A}}^{2} / \ln C_{\mathrm{A}}+R .
$$

A more rigorous theoretical argument for the form of the last three equations has been derived recently, ${ }^{26}$ also including particle correlation functions as well as higher order terms for higher densities and modifications for source term correlations.

\subsection{3. $A+B$ Reactions}

This class of reactions is the most abundant, important, intriguing, and challenging. The first demonstration that a completely segregated steady state can be achieved with a uniformly random source term ${ }^{42}$ was a real intellectual surprise. While the simulation demonstrations were performed on fractal lattices (Sierpinski gasket ${ }^{45}$ ) and critical percolation clusters, ${ }^{46}$ it turns out $^{27,41}$ that this phenomenon also occurs in one- and two-dimensional media, and, theoretically, even in three-dimensional media (however, only for infinite sys$\mathrm{tems}^{47}$ ). In addition, it has been shown ${ }^{42}$ that segregation occurs also for systems made of "walkers" (A) and "sitters" (B), though the distributions and the rate laws may differ. However, the most interesting aspect of steady-source reactions is the role of the source structure and statistics. The most subtle differences in the nature of the source may cause very dramatic differences in particle distributions and rate laws. An illustration of this is given in Table 2 (table I of Clement et al..$^{25}$ ). We see here how the segregation length $\Lambda$ is affected by the source conservation laws (strict vs. statistical), source correlation length $(\delta)$ and nature (random vs. fixed), by the presence of vertical annihilation, and by the pres- ence of a "negative" source term, such as desorption or natural decay (with first-order rate constant $K$ ). The reaction-diffusion parameters that enter the quantitative expression are the diffusion constant $(D)$, the source rate $(R)$, the particle size $(a)$, the lattice size $(L)$ and, of course, the dimensionality $(d)$.

\subsubsection{A + C Reactions}

It has already been shown a decade ago (Agranovich and Galanin ${ }^{16}$ ) that for a trapping reaction $\mathrm{A}+\mathrm{T} \rightarrow \mathrm{T}$ on a one-dimensional lattice, with a steady, uniformly random source of A particles, the trapping rate is linear in A density but quadratic in trap density. This is true for both random and even (superlattice) trap distributions (though the rate coefficients differ):

$$
\text { Rate } \sim C_{\mathrm{A}} C_{\mathrm{B}}^{2} \text {. }
$$

This result has also been derived via a somewhat different approach by Peacock-Lopez and Keizer. ${ }^{48}$ This nonclassical rate law is again due to a nonuniform $\mathrm{A}$ particle distribution.

Based on preliminary simulations ${ }^{49}$ and theoretical work, ${ }^{31}$ the last equation can be generalized in the following way:

$$
\begin{array}{ll}
\text { Rate }-C_{\mathrm{A}} C_{\mathrm{B}}^{2 / d_{\mathrm{s}}}, & d_{\mathrm{s}}<2 \\
\text { Rate }-C_{\mathrm{A}} C_{\mathrm{B}}, & d_{\mathrm{s}}>2,
\end{array}
$$

where $d$ is an effective spectral dimension and the marginal dimensionality is again two (at $d=2$ there is prob-

\begin{tabular}{|c|c|c|c|c|c|c|}
\hline Case $^{a}$ & $\begin{array}{c}\text { Conservation } \\
\text { conditions }\end{array}$ & Source case & & $d=1$ & $d=2$ & $d=3$ \\
\hline (a) & Strict & Random landing (random $\delta$ ) & $K=0$ & $L / 6$ & $a \ln L / a$ & $3 a$ \\
\hline (b) & Strict & Correlated landing (fixed $\delta$ ) & $K=0$ & $\delta$ & $a \ln \delta / a$ & $2 a$ \\
\hline (c) & Statistical & $\begin{array}{l}\text { Independent landing } \\
\text { Nonvertical annihilation } \\
\text { Vertical annihilation } \\
\text { Symmetric desorption }\end{array}$ & $\begin{array}{l}K=0 \\
K=0 \\
K \neq 0\end{array}$ & $\begin{array}{c}\text { saturation } \\
\xi^{\mathrm{c}} \\
\xi^{\mathrm{c}}\end{array}$ & $\begin{array}{c}\text { saturation } \\
a \ln \xi / a \\
a \ln \xi / a\end{array}$ & $\begin{array}{c}\text { saturation } \\
3 a \\
3 a\end{array}$ \\
\hline
\end{tabular}
ably again a logarithmic correction to eq $28 \mathrm{~b}$ ).

Table 2. Scaling Behavior of $\Lambda$, Typical Size of Segregated Domains, as a Function of the Euclidean Dimension $d$ and Various Source Conditions

${ }^{a}$ Cases (a) and (b) are, respectively, random and correlated landing ( $\delta$ is the separation between landing pairs) with conservation of the number of A's and B's on the system. For cases (c), the conservation condition is removed and a first-order decay mechanism is included (with a constant $K$ ). The system size is $L$, the size of a particle is $a$, and the external rate of arrival of particles is $R$.

'Saturation means that only one of the species occupies all the system.

cFor vertical annihilation: $\xi=\sqrt{D / a^{d} R}$; for symmetric desorption: $\xi=\sqrt{D / K}$. 


\section{EXPERIMENTS}

Examples of chemical reactions, photochemistry, and photophysics have been selected in order to illustrate the peculiarities of diffusion-controlled reaction kinetics in low dimensions.

\subsection{Photochemistry in Pores of Membranes}

The photodimerization of anthracene in solution was probably the first well-studied diffusion-limited reaction. ${ }^{50}$ The bimolecular reaction $\mathrm{A}+\mathrm{A} \rightarrow \mathrm{A}_{2}(\mathrm{~A}=\mathrm{an}$ thracene) followed the classical kinetic laws. A similar reaction provided the first study of fractal chemical kinctics:

$$
\mathrm{N}^{*}+\mathrm{N}^{*} \rightarrow \mathrm{N}_{2}^{* *} \rightarrow \mathrm{N}+\mathrm{N}+h v .
$$

Here $\mathrm{N}^{*}$ is a naphthalene molecule excited to its first triplet state and $\mathrm{N}_{2}^{* *}$ is the transitory dimer in the excited first singlet state. ${ }^{5,6}$ The experiment is carried out in a solution embedded in various porous membranes (nylon, acetate, paper; see Fig. 4). The naphthalene molecules diffuse inside the solvent inside the pores.

Figure 5 shows the pores of some of the channel-pore membranes used as "test-tubes." These cylindrical tubes are $6000 \mathrm{~nm}$ long and have radii ranging from 1000 to $10 \mathrm{~nm}$. Typical molecular diffusion lengths in these experiments are on the order of $100 \mathrm{~nm}$. We thus expect to see a range of kinetic behaviors, from three-dimensional to one-dimensional, depending on the molecular diffusion parameters. Indeed, ${ }^{5}$ the heterogeneity exponent $h$ (see eq 9) ranges from $h=0.07$ for $1000-\mathrm{nm}$ pores to $h$ $=0.41$ for $25-\mathrm{nm}$ pores. This is in good agreement with the theoretical limits, i.e., $h=0$ for three-dimensional media and $h=1 / 2$ for one-dimensional media. Moreover, for an intermediate pore radius $(100 \mathrm{~nm})$ one observes a crossover in time, from $h=0.06$ in the millisecond regime to $h=0.48$ in the subsecond regime.

Earlicr experiments, ${ }^{6}$ carried out in ordinary porous membranes made of nylon (Fig. 4), acetate, or paper give $h$ values in the range of 0.3 to 0.4 . This can be interpreted simply as some statistical averaging over various pore domains ranging between one-dimensional and three-dimensional topologies. Alternatively, one can ascribe to such membranes "effective" spectral dimensions $d_{3}$ (see eq 10), with values between 1.2 and 1.6.

\subsection{Photophysics in Polymer Blends}

Excitation kinetics in molecular aggregates and materials is of biological interest (e.g., photosynthesis and neuron transmission ${ }^{11,12}$ ), morphological interest (e.g., studies of polymeric materials ${ }^{7-9}$ ), and potential technological interest (nano-devices) ${ }^{51}$ ). Both $\mathrm{A}+\mathrm{A}$ and

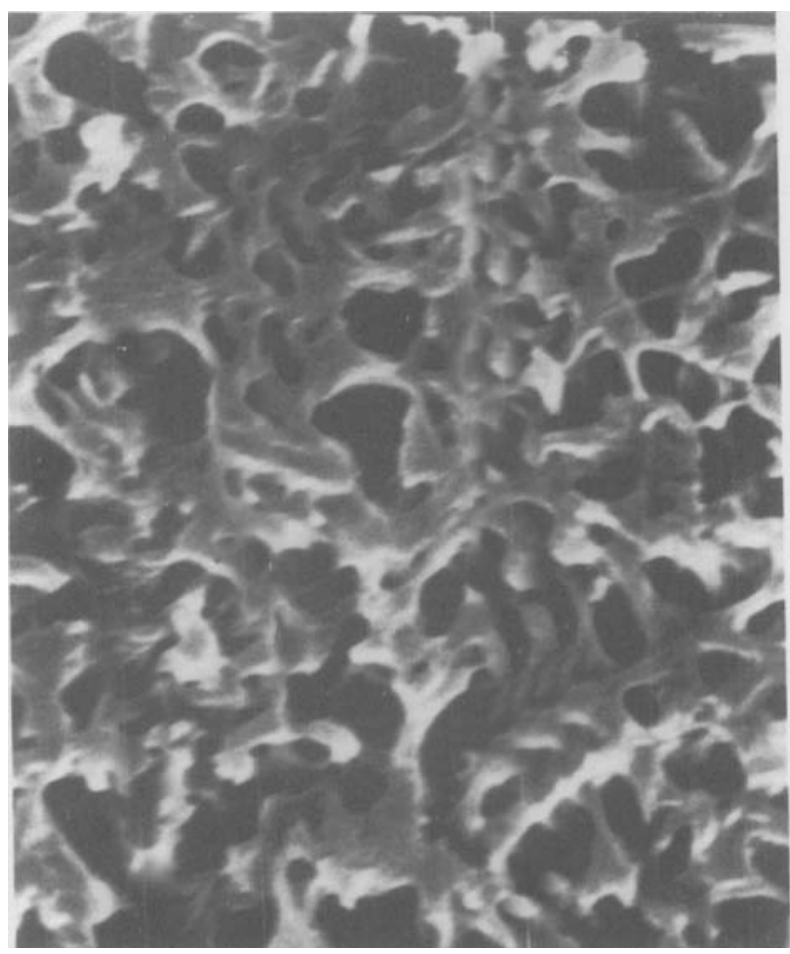

Fig. 4. Porous nylon membrane (Gelman, Ann Arbor, MI, USA). Magnification about $10^{4}$.

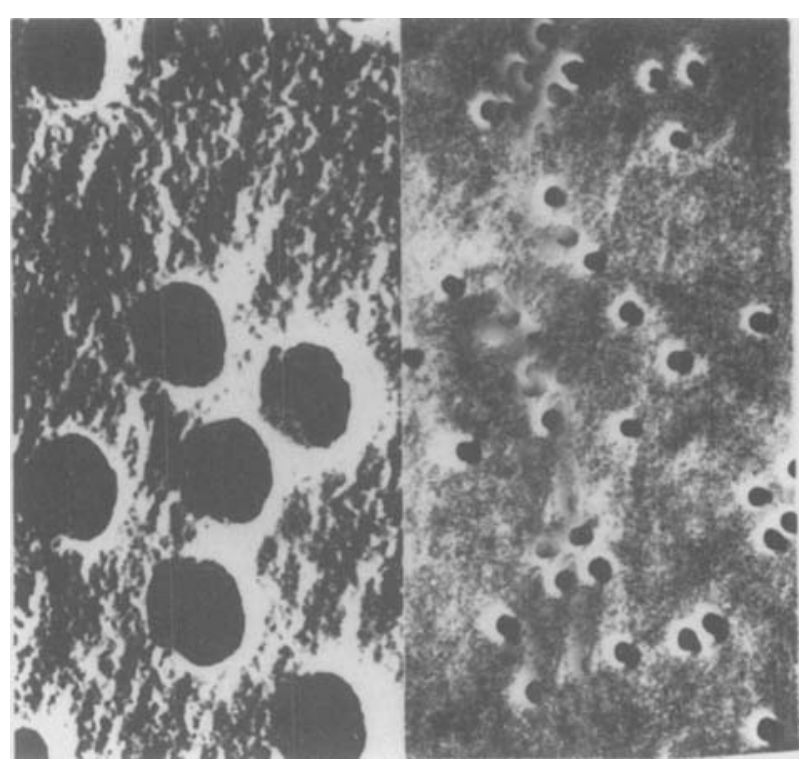

Fig. 5. Polycarbonate channel-pore membranes. (Nuclepore, Pleasanton, CA, USA). Magnification about $10^{4}$. Channel length is $6 \mu \mathrm{m}$ and the radius of the channels on the left is $1 \mu \mathrm{m}$ while that on the right is $0.1 \mu \mathrm{m}$. 
$A+C$ type reactions have been studied under tightly controlled conditions using an extremely dilute blend of poly-1-vinyl-naphthalene (PIVN) in polymethyl-methacrylate (PMMA). The electronic excitation is confined to the naphthalene moieties of the P1VN. Triplet excitations are utilized because of their limited range of energy transfer ( 4 to $8 \AA$ ). In the absence of neighboring P1VN chains, the excitation transport is thus limited to a single chain and reflects its one-dimensional topology. We therefore limit ourselves to the very dilute blends (about $0.01 \%$ P1VN or less) where the P1VN chains are effectively isolated from each other.

\subsection{1. $A+A \rightarrow$ Product Reaction (Homofusion)}

With short pulse laser excitations ${ }^{9}$ the observed phenomenon is that of triplet-triplet fusion ("annihilation"):

$$
\mathrm{T}+\mathrm{T} \rightarrow \mathrm{S} \text {. }
$$

The singlet excitations (S) have a natural lifetime of about 100 ns and are monitored via the resultant fluorescence (UV), while the triplet excitations (T) have a natural lifetime of about $3 \mathrm{~s}$ and are monitored via their phosphorescence (green).

For the most dilute samples measured (e.g., 0.05\%), the heterogeneity exponent was found to be about 0.4 , thus approaching the theoretical expectation, $h=1 / 2$, for one-dimensional confinement of the reaction kinetics. On the other hand, for pure (100\%) P1VN material the experimental result is $h=0.02 \pm 0.02$, in good agreement with the expectation for three-dimensional (classical) systems $(h=0)$.

\subsection{2. $A+C \rightarrow C+$ Products Reaction (Heterofusion)}

Under prolonged UV excitation of P1VN blends, excimers are produced, ${ }^{7-9}$ both in the singlet and the triplet states. The excimers are essentially defect sites with trapped excitations. The mechanism of triplet-triplet an- nihilation can now be written, to a good approximation $^{7,8}$ as:

$$
\mathrm{T}_{\mathrm{F}}+\mathrm{T}_{\mathrm{E}} \rightarrow \mathrm{T}_{\mathrm{E}}
$$

where $T_{F}$ is the free triplet excitation and $T_{E}$ the trapped excimer excitation. The excimer sites are thus "catalytic" sites where the free excitations are "trapped" and consumed in an annihilative reaction.

From eqs 8 to 10 we expect the heterogeneity exponent $h=1 / 2$ for $d=1$ and $h=0$ for $d=3$, in agreement with the exact analytical results ${ }^{30}$ for binary reaction kinetics (eqs 22 and 23). Measurements have been conducted for very dilute blends ${ }^{9}(0.01 \%$ and $0.005 \%)$ and the average result is $h=0.50 \pm 0.03$, as expected for truly one-dimensional reaction topologies.

\subsection{Bimolecular Reaction in Capillary with Gel}

The chemical reactions were performed in a rectangular or a square glass capillary. The binary complex reaction

$$
\begin{gathered}
\mathrm{Cu}^{2+}+\text { disodium ethyl bis(5-tetrazolylazo)acetate } \\
\text { trihydrate } \rightarrow 1: 1 \text { complex }
\end{gathered}
$$

was carried out in a gel inside the glass tube, allowing efficient diffusion but little or no convection. At time 0 , there is a sharp boundary between $A$ and $B$, and that is where product $\mathrm{C}$ is being formed (see Fig. 3). The absorbance of the product $\mathrm{C}$ was monitored at every fixed period by moving a detector (PMT) and a light source (green light-emitting diode), which are fixed on a stepping motor, along the reaction front domain, while the reactor is fixed in time.

The results are summarized in Table 3. These results agree well with the theoretical expectations $(\alpha=1 / 2$, $\beta=1 / 6, \delta=1 / 2$ ) given in Section 2.1.5.

Acknowledgment. This work was supported by NSF grant DMR 88-01120.

Table 3. Time Exponents from Experiments

\begin{tabular}{lcrccc}
\hline Reactor size & $C_{\mathrm{Cu}}$ & \multicolumn{1}{c}{$C_{\text {erra }}$} & $\alpha$ & $\beta$ & $\delta$ \\
\hline $4 \times 2 \mathrm{~mm}$ & $10^{-3} \mathrm{M}$ & $5 \times 10^{-5} \mathrm{M}$ & 0.48 & 0.16 & 0.53 \\
& $10^{-3} \mathrm{M}$ & $7.5 \times 10^{-5} \mathrm{M}$ & $0.53,0.48$ & $0.15,0.20$ & $0.51,0.54$ \\
& $2 \times 10^{-3} \mathrm{M}$ & $6 \times 10^{-5} \mathrm{M}$ & 0.52 & 0.16 & 0.53 \\
$2 \times 2 \mathrm{~mm}$ & $2 \times 10^{-3} \mathrm{M}$ & $6 \times 10^{-5} \mathrm{M}$ & 0.60 & 0.22 & 0.52 \\
\hline
\end{tabular}

$\mathrm{Cu}$ is $\mathrm{Cu}^{2+}$ and tetra is disodium ethyl bis(5-tetrazolylazo)acetate trihydrate, where the solvent is $0.15 \%$ agarose/water. $C_{\mathrm{Cu}}$ and $C_{\mathrm{tetr}}$ are $t=0$ concentrations. Here $x_{\mathrm{f}} \sim t^{\alpha}, w-t^{\beta}$, and $R-t^{-\delta}$. 


\section{REFERENCES}

(1) De Gennes, P.G. J. Chem. Phys. 1982, 76: 3316.

(2) Havlin, S.; Ben-Avraham, D. Adv. Phys. 1987, 36: 695; Rudnick, J.; Gaspari, G. Science 1987, 237: 384.

(3) (a) Argyrakis, P.; Kopelman, R. J. Chem. Phys. 1980, 72: 3053. (b) Klymko, P.W.; Kopelman, R. J. Phys. Chem. 1983, 87: 4565.

(4) Kopelman, R. Science 1988, 241: 1620.

(5) Prasad, J.; Kopelman, R. Chem. Phys. Lett. 1989, 157 535.

(6) Prasad, J.; Kopelman, R.J. Phys. Chem. 1987, 91: 265.

(7) Li, C.-S.; Kopelman, R. Macromolecules 1990, 23: 2223.

(8) Li, C.-S.; Kopelman, R.J.Phys. Chem. 1990, 94: 2135.

(9) Shi, Z.-Y.; Li, C.-S.; Kopelman, R. In Polymer Based Molecular Composites; Mark, J.E.; Schaefer, D.W., Eds.; Proceedings of Materials Research Society: Pittsburgh, 1989.

(10) Drake, J.M.; Levitz, P.; Turro, N.J.; Nitsche, K.S.; Cassidy, K.F. J. Phys. Chem. 1988, 92: 4680.

(11) Fauman, E.B.; Kopelman, R. Comments Mol. Cell. Biophys. 1989, 6: 47.

(12) Dissado, L.A. Comments Mol. Cell. Biophys. 1987, 4: 143.

(13) Hoshen, J.; Kopelman, R. J. Chem. Phys. 1976, 65: 2817.

(14) Klymko, P.; Kopelman, R. J. Phys, Chem. 1982, 86: 3686.

(15) Ovchinikov, A.A.; Zeldovich, Ya.B. Chem. Phys. 1978, 28: 215, and references cited therein. (b) Kuzovkov, V.N.; Kotomin, E.A. J. Phys. C 1984, 17: 2283.

(16) Agranovich, V.M.; Galanin, M.D. Electronic Excitation Energy Transfer in Condensed Matter; North Holland: Amsterdam, 1982.

(17) (a) Smoluchowski, M.V.Z. Phys. Chem. 1917, 92: 129. (b) Keizer, J. J. Phys. Chem. 1982, 86: 5052, and references cited therein.

(18) Klafter, J.; Blumen, A; Zumofen, G. J. Stat. Phys. 1984, 36: 561 .

(19) Kopelman, R. J. Stat. Phys. 1986, 42: 185.

(20) Kang, K.; Redner, S. Phys. Rev. Lett. 1984, 52: 955.

(21) Alexander, S.; Orbach, R. J. Phys. (Paris), Lett. 1982, 43: L625.

(22) Blumen, A.; Klafter, J.; Zumofen, G. In Optical Spectroscopy of Glasses; Zschokke, I., Ed.; Reidel: Dordrecht, 1986; p. 199.

(23) Lindenberg, K.; West, B.J.; Kopelman, R. In Proceedings of Conference on Noise and Chaos in Nonlinear Dynamical Systems; Catelin, S.; Moss, F., Eds.; Cambridge University Press: London, 1990; p. 142.

(24) Clement, E.; Sander, L.M.; Kopelman, R. Phys. Rev. A 1989, 39: 6455 .
(25) Clement, E.; Sander, L.M.; Kopelman, R. Phys. Rev. A 1989, 39: 6466.

(26) Clement, E.; Sander, L.M.; Kopelman, R. Phys. Rev. A 1989, 39: 6472.

(27) Harmon, L.A.; Li, L.; Anacker, L.W.; Kopelman, R. Chem. Phys. Lett. 1989, 163: 463.

(28) Parus, S.J.; Kopelman, R. Phys. Rev. B 1989, 39: 889.

(29) Argyrakis, P.; Kopelman, R. Phys. Rev. A 1990, 41: 2114; Phys. Rev. A 1990, 41: 2121.

(30) Weiss, G.; Kopelman, R.; Havlin, S. Phys. Rev. A 1989, 39: 466.

(31) Clement, E.; Kopelman, R.; Sander, L.M. Europhys. Lett. 1990, 11(8): 707.

(32) Doering, C.R.; Ben-Avraham, D. Phys. Rev. A 1988, 38: 3035 .

(33) Ben-Avraham, D.; Doering, C.R. Phys. Rev. Lett. 1989, 62: 2563.

(34) Tomey, D.C.; McConnell, H.M. J. Phys. Chem. 1983, 87: 1441.

(35) Anacker, L.W.; Parson, R.P.; Kopelman, R. J. Phys. Chem. 1985, 89: 4758.

(36) Bramson, M.; Griffeath, D. Ann. Probab. 1980, 8: 183.

(37) Toussaint, D.; Wilczek, F. J. Chem. Phys. 1983, 78: 2642.

(38) Zumofen, G.; Blumen, A.; Klafter, J. J. Chem. Phys. 1985, 83: 3198 .

(39) Lindenberg, K.; West, B.J.; Kopelman, R. Phys. Rev. A 1990, 42: 890.

(40) Li, L. Ph.D. Thesis; University of Michigan: Ann Arbor, 1989.

(41) Galfi, L.; Racz, Z. Phys. Rev. A 1988, 38: 3151.

(42) Anacker, L.W.; Kopelman, R. Phys. Rev. Lett. 1987, 58: 289; J. Phys. Chem. 1987, 91 : 5555.

(43) Lindenberg, K.; West, B.J.; Kopelman, R. Phys. Rev. Lett. 1988, 60: 1777.

(44) Racz, Z. Phys. Rev. Lett. 1985, 55: 1707.

(45) Mandelbrot, B.B. Fractal Geometry of Nature; Freeman: San Francisco, 1983.

(46) Newhouse, J.S.; Kopelman, R. J. Chem. Phys. 1986, 85: 6804; J. Phys. Chem. 1988, 92: 1538.

(47) West, B.J.; Kopelman, R.; Lindenberg, K. J. Stat. Phys. 1989, 54: 1429.

(48) Peacock-Lopez, E.; Keizer, J. J. Chem. Phys. 1988, 88: 1997.

(49) Anacker, L.W.; Kopelman, R. In Science at the John von Neumann National Supercomputer Center, 1989; Cook, G., Ed.; Consortium for Scientific Computing: Princeton, NJ, 1990; p. 29.

(50) Luther, R.; Weigert, F. Z. Phys. Chem. 1905, 53: 385.

(51) Lieberman, K.; Hanush, S.; Lewis, A.; Kopelman, R. Science 1990, 247: 59. 